\title{
Numerical simulation and experimental calibration of Additive Manufacturing by blown powder technology. Part I: thermal analysis
}

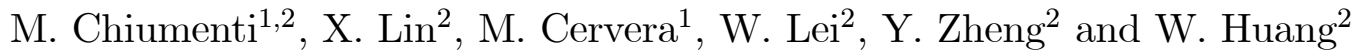 \\ ${ }^{1}$ International Center for Numerical Methods in Engineering (CIMNE) \\ Universidad Politécnica de Cataluña (UPC) \\ Edificio C1, Campus Norte, Gran Capitán s/n, 08034 Barcelona, Spain. \\ e-mail: michele@cimne.upc.edu, web page: http://www.cimne.com \\ ${ }^{2}$ State Key Laboratory of Solidification Processing (SKLSP), \\ School of Material Sciences and Engineering, Northwestern Polytechnical University (NWPU), \\ 710072, Xi'an, China.
}

KEYWordS: Additive Manufacturing (AM) process, Metal Deposition (MD) process, blown powder technology, powder feeding, Laser Solid Forming (LSF), FE modeling, thermo-mechanical analysis.

\begin{abstract}
In this work the numerical simulation of Additive Manufacturing (AM) processes is addressed. The numerical results are compared with the experimental campaign carried out at SKLSP laboratories, where a Laser Solid Forming (LSF) machine, also referred to as Laser Engineered Net Shaping (LENS), is used to fabricate metal parts directly from CAD models. Ti-6Al-4V metal powder is injected into the molten pool created by a focused, high-energy laser beam and a layer of added material is sinterized according to the laser scanning pattern specified by the user.

The objectives of this paper are twofold: firstly, this work is intended to calibrate the software for the numerical simulation of the AM process, to achieve high accuracy. Secondly, the sensitivity of the numerical model to the process parameters and modelling data is analysed.

The numerical model adopts an apropos FE activation technology, which reproduces the same scanning pattern set for the numerical control system of the AM machine. This consists of a complex sequence of polylines, used to define the contour of the component, and hatches patterns to fill the inner section. The full sequence is given through the Common Layer Interface (CLI) format, a standard format for different manufacturing processes such as Rapid Prototyping (RP), Shape Metal Deposition (SMD) or machining processes, among others. The result is a layer-by-layer metal deposition which can be used to build-up complex structures for components such as turbine blades, aircraft stiffeners, cooling systems, or medical implants, among others.
\end{abstract}




\section{Introduction}

Additive Manufacturing (AM), also known as 3D printing or Rapid Prototyping (RP), is a relatively novel technique to make parts, layer upon layer, directly from 3D model data. It is the opposite of subtractive manufacturing processes such as machining. 3D printing means that the fabrication makes use of print-heads or nozzles among other printing technologies.

This work focuses on the AM technologies for Metal Deposition (MD) processes by melting and sintering metal powders (e.g. titanium, Inconel, steel) making use of high-energy sources such as Electron Beam or laser.

The key benefit of the AM process is the freedom of design allowing for complex shapes, inner cavities impossible to produce by machining, very thin walls difficult to produce by casting, light weight parts with lattice/foam inner structures. The process flexibility allows for customized design in short production time where multiple pieces can be built as one without the need of complex tooling (e.g. moulds). Furthermore, another advantage of this process consists of the rapid cooling of each deposited layer and, consequentially, a finer grain size compared to other metal forming technology such as casting or forming.

A large number of products can be created using the AM technology: turbine blades, aircraft stiffeners, jet engine parts, microturbines, fuel injection swirler are some examples in the aeronautical sector. Brackets, hydraulic manifolds, cooling systems, boat propellers, heat shields and different tooling inserts are some product in the automotive sector. Dental bridges as well as many different implants for the medical surcery and even a laroe variety of conserner coods oan be alo

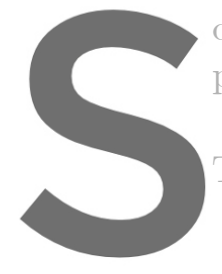
produced using this te

There are different

They can be classified

1. Powder bed tech

Laser Sintering (SLS), where the metal powder is melted by a laser beam. Electron Beam

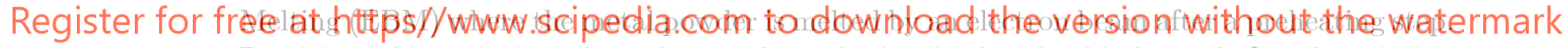

Precision inkjet printing where the metal powder is mixed with a binder and after the printing operation, the part is consolidated by sintering. The main features of these technologies are the possibility of working with complex shapes, including small internal cavities thin walls, and a high-quality surface finishing.

2. Blown powder or powder feeding technologies, also known as Laser Metal Deposition or Laser cladding. In this process, the metal powder is blown coaxially to the laser beam which melts the particles on a substrate to form a metallurgical bond when cooled. Laser Engineered Net Shaping (LENS) or Laser Solid Forming (LSF), makes use of this technology allowing for higher powder deposition rates compared to the powder bed technologies as well as the manufacturing of larger parts using coarser metal powders ([36], [29], [30] and [20]).

The FE method has been used to study metal deposition processes and different kind of additive manufacturing technologies, often taking advantage from the experience acquired in modeling welding processes ([2], [24], [21], [22], [37], [35], [10], [3], [26], [14], [23] and [19]).

In this work the Additive Manufacturing process by blown powder technology is addressed. On the one hand, the FE framework to deal with the analysis of the AM process is presented. The 
description of the activation technique necessary to simulate the layer-by-layer metal deposition is detailed. On the other hand, an exhaustive experimental campaign is carried out at the State Key Laboratory of Solidification Processing (SKLSP) using a LSF machine. Ti-6Al-4V powder is the reference material used to produce the samples for the calibration and sensitivity analysis of the numerical model.

The expected outcomes of this work are twofold: firstly, the experimental campaign is intended as a reference for the numerical analysis to capture the material response in terms of either temperature or displacement evolution at different locations during the full duration of the AM process and the following cooling phase. Secondly, the confidence on the predictive accuracy of model calculations is used to design more and more complex scanning patterns minimizing distortions, residual stress and hot-cracking risk.

\section{Heat transfer analysis}

Both the heating and the cooling phases of the AM process are controlled by the balance of energy equation. This can be stated as:

$$
\dot{H}=-\nabla \mathbf{q}+\dot{Q}+\dot{D}_{\text {mech }}
$$

where $\dot{H}$ is the enthalpy rate (per unit of volume), q is the heat flux, while $\dot{Q}$ and $\dot{D}_{\text {mech }}$ represent the heat source (per unit of volume) and the thermo-mechanical dissipation rate (per unit of the hos volume), respectively.

For the AM proces formed along the scanning path dissipation can be neglected in

The enthalpy $H(T$ liquid fraction, $f_{L}$. Hence, the enthalpy rate in
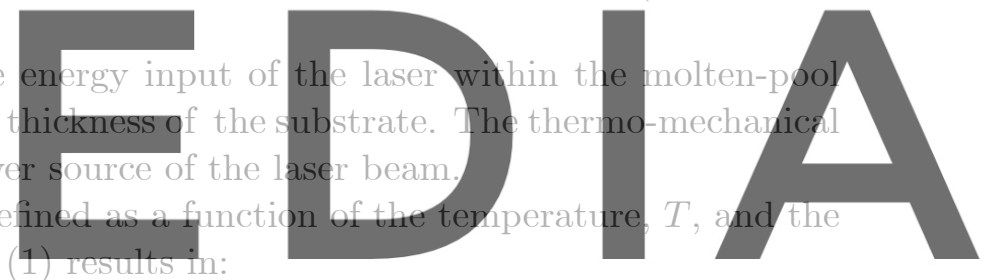

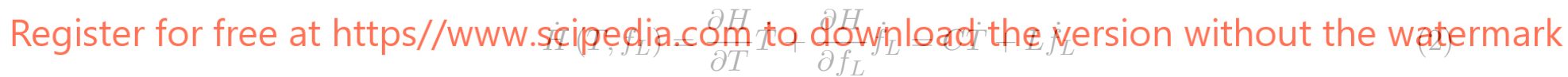

where $C(T)=\frac{\partial H}{\partial T}$ is the (temperature dependent) heat capacity and $L=\frac{\partial H}{\partial f_{L}}$ is the latent heat released during the phase-change process.

The heat capacity is usually defined as: $C=\rho c$, the product of the material density, $\rho$, and the specific heat, $c$.

During the phase transformation the material volume, $V$, can be split into liquid and solid phases as: $V=V_{L}+V_{S}$. The liquid and solid fractions are defined as: $f_{L}=\frac{V_{L}}{V}$ and $f_{S}=\frac{V_{S}}{V}$, respectively, so that: $f_{L}+f_{S}=1$. The evolution of the liquid fraction $\dot{f}_{L}$ or, alternatively, $\dot{f_{S}}=-\dot{f}_{L}$ defines the phase change, that is, how the latent heat is absorbed or released during the transformation.

Remark 1 On the one hand, the energy input induces the fusion of the material in the moltenpool followed by the solidification process which produces the coalescence of the metal powder on the substrate forming the new layer. On the other hand, the energy balance (accounting for the energy absorbed during the melting and the energy released during the solidification) is null and very localized in the Heat Affected Zone (HAZ). 
Remark 2 The amount of latent heat is negligible in front of the energy input coming from the laser. Moreover, the HAZ is very narrow and the phase change occurs very fast compared to the thermal diffusion process, so that its global effect is minor in the heat transfer analysis.

The heat flux (per unit of surface) q, is computed as a function of the temperature gradient through the Fourier's law as:

$$
\mathbf{q}=-k \nabla T
$$

where $k(T)$ is the (temperature dependent) thermal conductivity. Due to the high conductivity of the metallic materials, the thermal diffusion process is the key mechanism of the metal deposition, driving the heat transfer in the manufacturing process.

Let $V$ be an open and bounded domain in $\mathbb{R}^{n_{\operatorname{dim}}}$ where $n_{\operatorname{dim}}$ is the number of dimensions of the space, closed by the smooth boundary $S=S_{T} \cup S_{q}$ where the corresponding boundary conditions are defined in terms of either prescribed temperature on $S_{T}$ or prescribed heat flux on $S_{q}$, being $S_{T} \cap S_{q}=0$. Suitable initial conditions for the transient thermal problem are defined in terms of initial temperature field: $T(t=0)=T_{0}$.

The resulting weak (integral) form of the energy balance equation (1), used for the heat transfer analysis, can be written as:

$$
\int_{V}\left[\left(\rho c \dot{T}+L \dot{f}_{L}\right) \delta T\right] d V+\int_{V}[k \nabla T \cdot \nabla(\delta T)] d V=W_{\text {ther }}^{\text {ext }} \quad \forall \delta T
$$

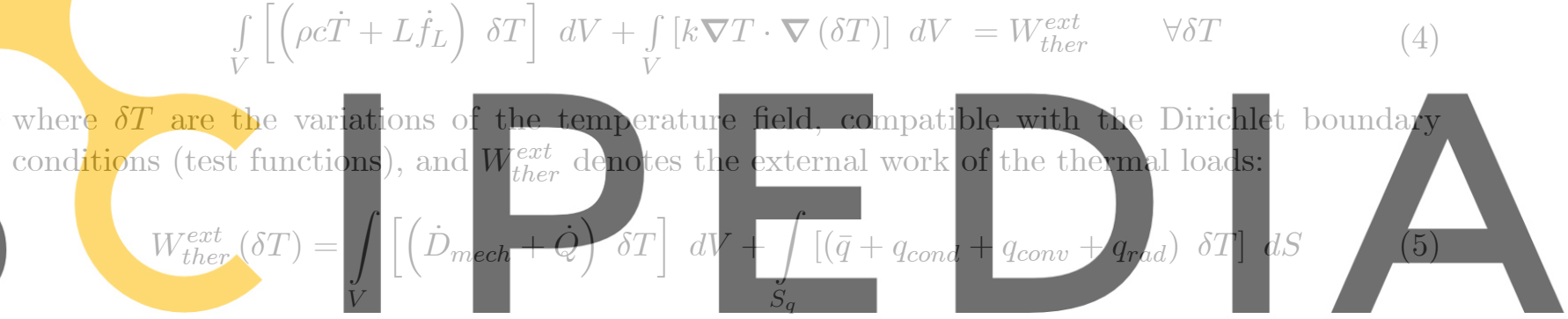

The prescribed heat flux, $\bar{q}$, defined in (5), can be used to spread part of power input over the

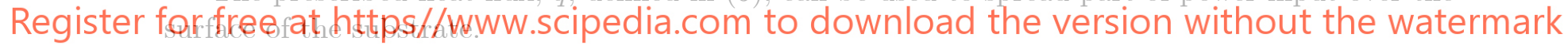

The heat loss by convection, qconv, can be taken into account using the Newton's law as:

$$
q_{\text {cond }}=h_{\text {conv }}\left(T-T_{\text {env }}\right)
$$

where $h_{\text {conv }}(T)$ is the (temperature dependent) Heat Transfer Coefficient (HTC) by convection, while $T$ and $T_{e n v}$ are the temperatures of material surface and the surrounding environment, respectively (see [9]).

Remark 3 It is common to assume a constant value for the temperature of the surrounding environment, assuming that the process does not affect its value. This is not the case when the AM process is carried out in a close chamber in a controlled atmosphere (e.g. argon to prevent the surface oxidation). The duration of the process is generally very long and a lot of energy is scattered in the chamber. The result is an increment of the environment temperature that cannot be neglected.

Similarly, the heat flux due to the heat conduction process between the component and the clamping system, $q_{\text {cond }}$, can be computed as:

$$
q_{\text {cond }}=h_{\text {cond }}\left(T-T_{\text {clamp }}\right)
$$


where $h_{\text {cond }}(T)$ is the (temperature dependent) HTC by conduction between the two materials in contact, and $T_{\text {clamp }}$ is the temperature of the clamping system.

Remark 4 The HTC by conduction is defined as the inverse of the thermal resistivity between the two material in contact and it depends on different parameters defined at the contact interface such as the contact pressure and the surface roughness, among others. Considering contact between metallic materials, the value of the HTC is very high, around $1000\left[\mathrm{~W} / \mathrm{m}^{2} \mathrm{~K}\right]$. Hence, Newton's law can be replaced by prescribing the temperature at the corresponding contact surface, $S_{T}$ as: $T=T_{\text {clamp }}$.

Remark $5 T_{\text {clamp }}$ represents the temperature of the clamping system used to hold the substrate where the AM process takes place. Also in this case, its value cannot be assumed as constant in time if the thermal inertia of the substrate is comparable to the one of the clamping system (this was the case in our work). In fact, the high temperatures registered in the substrate are transmitted to the clamping system through the contact interface between them. This interface exhibits a very low thermal resistance to the heat flux when two metallic components are pressed together. Hence, the value of $T_{\text {clamp }}$ should be known (or estimated) to avoid of including the clamping system as part of the thermal analysis.

Finally, the radiation heat flux, $q_{\text {rad }}$, is the most important condition to dissipate the heat of the molten-pool and, more generally, at the HAZ, due to the high temperature field induced by the heat source. The radiation heat flux can be computed using Stefan-Boltzmann's lav as:
where $\sigma_{\text {rad }}$ is the Stefan-Boltznann constant and $\varepsilon_{\mathrm{rad}}$ is the emissivity paraneter, respectiyely.

Remark 6 Stefan-Boltzmann's law can be rewritten as:

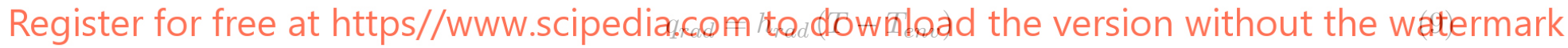

where $h_{\text {rad }}(T)$ is the (temperature dependent) HTC by radiation defined as:

$$
h_{\text {rad }}(T)=\sigma_{\text {rad }} \varepsilon_{\text {rad }}\left(T^{3}+T^{2} T_{e n v}-T T_{e n v}^{2}-T_{e n v}^{3}\right)
$$

This format is interesting for two reasons: firstly, because it is possible to linearize the contribution of the heat radiation term as:

$$
q_{\text {rad }}=h_{\text {rad }}\left(T^{n}\right)\left(T^{n+1}-T_{\text {env }}\right)
$$

where $T^{n+1}=T\left(t^{n+1}\right)$ is the current temperature at time $t^{n+1}$, and and $T^{n}=T\left(t^{n}\right)$ is temperature in the previous time-step. The second reason is that it is extremely difficult to separate the heat losses due to convection or radiation mechanisms. Hence, the numerical model can assume a unique HTC accounting for both heat convection and radiation terms, as:

$$
q_{\text {loss }}=h_{\text {loss }}\left(T-T_{\text {env }}\right)
$$

where $h_{\text {loss }}(T)$ is the (temperature dependent) HTC accounting for the total heat loss through the surrounding environment. 


\section{FE modeling of the AM process}

The AM process is modeled by moving the heat source along a user-defined scanning pattern. In addition, the sintering process must be considered, which transforms the metal powder, injected into the molten-pool, into a new layer of material.

The numerical simulation of this process requires an $a d$-hoc procedure to apply the (volumetric) heat source (focussed on the substrate) to those elements affected by the moving energy input as well as to activate the elements forming the sinterized layer (which is advancing following the powder deposition). Therefore, at each time-step of the simulation, a searching algorithm is used to identify the elements within the molten-pool as well as those representing the advancing metal deposition layer. This activation strategy, referred to as born-dead-elements technique, classifies the elements defined in the original finite element mesh into: active, inactive and activated elements. On the one hand, the active elements (o.g. the mesh which define the substrate) are computed and assembled into the global matrix. On the other hand, the inactive elements, such as the entire discretized/domain where the scanning path is defined, are not included as part of the model: they have been generated but do not play any role in the computational model. At each time-step a number of elements (activated elements) are switched on according to the powder deposition along the scanning sequence defined by the user. Only active and activated elements are assembled into the solution model (see also [10]).

The advantage of this activation technique is the possibility of defining the computational mesh

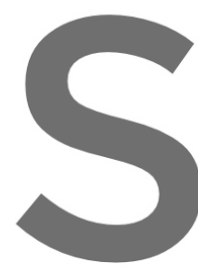
independently from th a Cartesian grid discre generation which coinc

the inner structures (hatch scanning) to obtain

Remark 7 The quiet element technique is a
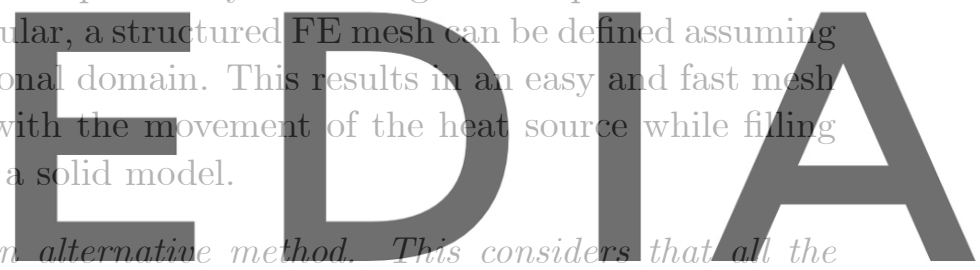

elements of the mesh defining the successive layers, which will be deposited during the process

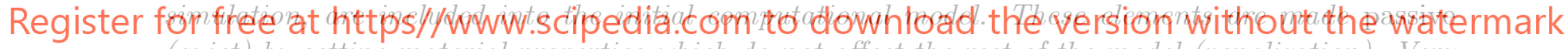
(quiet) by setting material properties which do not affect the rest of the model (penalization). Very low values for both heat capacity and thermal conductivity are considered. Later on, following to the metal deposition process, the elements are activated, meaning that their real thermo-physical properties are re-established ([19]). This approach has different drawbacks: firstly, the simulation process is performed assembling all the elements and solving the full system of equations leading to a very high computational cost. Secondly, the penalization of the material properties of the passive elements induces an ill-conditioning of the solution matrix which may cause numerical problems for its solution such as a reduction of the convergence ratio of iterative solvers. Furthermore, when the focus is the stress analysis, fictitious strains and temperature gradients are accumulated in the quiet elements and they will be transformed into spurious stresses and heat fluxes when these elements are activated, spoiling the solution.

One of the added features of this work is the possibility of specifying the position of the laser source using the same input data as for the process machine. The scanning-path is defined by a Common Layer Interface (CLI) format described in the following section. This is a great advantage, which simplifies the end-user work integrating the machine directives with the software interface. Note that the scanning-path only define the sequence of points along the scanning trajectory as 
well as the reference plane where the energy beam is focussed. There are no references neither to the size of the molten-pool nor to the thickness of the deposited layer. The section of the layer deposited; that is, its thickness and width, as well as the spot-size of the laser beam must be specified to inform properly the simulation process.

Remark 8 The objective of the present work is the analysis of the AM process at global level (component scale) where the size of the layer section is assumed as known. It is out of the scope of this work the study of the process at local level (molten-pool analysis), which characterizes the relationship among the different process parameters affecting the powder sintering process as: the energy input affecting the temperature of the molten-pool and the surrounding HAZ (see [33] and [34]), the laser-spot size, the scanning speed, the powder feeding (flow-rate, etc..), etc.

From the numerical point of view, the implications derived from the space discretization (FE mesh adopted) and the time-stepping defined for the time-integration of the thermal (transient) problem must be considered.

A first consideration regards the discretization in time. The discrete problem is characterized by a time-step, $\Delta t=t^{n+1}-t^{n}$, so that a discrete sequence of computations is performed according to the time-marching scheme. Therefore, the molten-pool does not continuously move along the scanning path (as for the continuous problem) but, instead, it is stepping from its position at time $t^{n}$ to that at time $t^{n+1}$. Consequently, the power input is intermittent along the scanning path, producing a discontinuous HAZ. The problem can be alleviated by reducing the time-step, to get an overlapped molten Alternatively, the the elements belongin at both ends the molten-pools rectangular section $v_{M D} \Delta t$ long (being $v_{M D}$ th
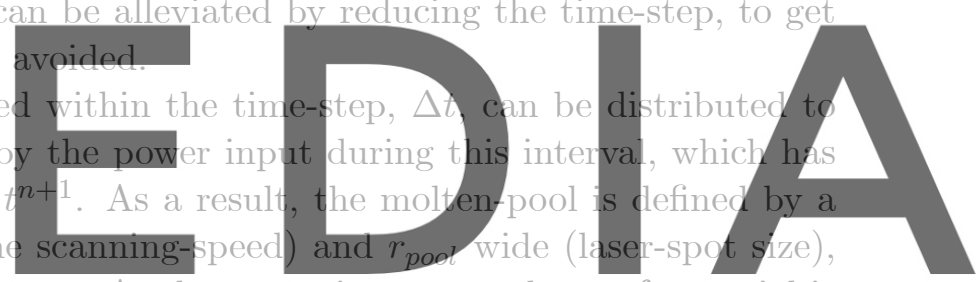

which penetrates $h_{\text {pool }}$ in depth within the substrate. At the same time, a new layer of material is

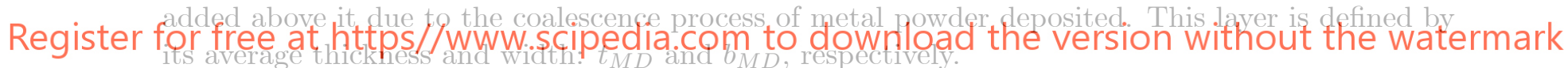

A second consideration is about the space discretization. Even if the HAZ is discretized using a very fine mesh, it is very challenging to achieve enough spatiai resolution to define exactiy the ideal volume where the power input must be delivered.

Remark 9 In the literature it is possible to find different models to represent the heat source with different degrees of sophistication. The double ellipsoidal power density distribution illustrated by Goldak et al. ([18]) is the base of many models proposed for its accurate characterization (e.g. [28], [31], [16], [27] and [25]). This is mandatory, when the focus is the local-level analysis to study the thermal convection within the welding-pool. Nevertheless, at global-level, it is much more important to account for the right amount of energy introduced in the HAZ, more than its local density distribution.

According to the outcome of the searching algorithm, it is possible to sum the volume of all the elements belonging to the (discretized) molten-pool as:

$$
V^{\text {pool }}=\left.\sum_{e=1}^{n e} V^{(e)}\right|_{e \in \text { pool }}
$$


so that the (average) density distribution of the heat source (per unit of volume) is computed as:

$$
\dot{Q}=\frac{\eta \dot{P}}{V^{p o o l}}
$$

where $\dot{P}$ is the total energy input and $\eta$ the actual heat absorption efficiency. This power redistribution preserves the total energy input independently of the mesh used (see [10]).

The balance of energy equation in (4) establishes the equilibrium between the energy input and the heat loss. The same care devoted to estimate the energy delivered by laser beam and absorbed by the substrate must be placed to compute the heat dissipated through the boundaries of the AM domain. This domain is also changing due to the AM process, requiring a specific searching strategy to update the contour surface at each time-step of the numerical simulation. Following the activation process, this updating is necessary to compute the current size of the boundary surfaces subjected to the heat radiation and heat convection fluxes, and, consequently, perform an accurate heat transfer analysis of the AM process.

\section{CLI-format to define the scanning-path}

The Common Layer Interface (CLI) is a universal format for the input of geometry data to model fabrication systems based on layer manufacturing technologies (LMT). It is intended as a simple,

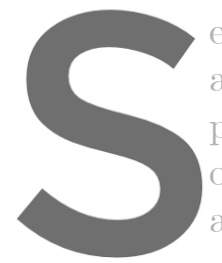
efficient and unambigu
allows the format to
photo-curing of resin
of powder materials
and machining process which build models on a layer-by-layer basis.
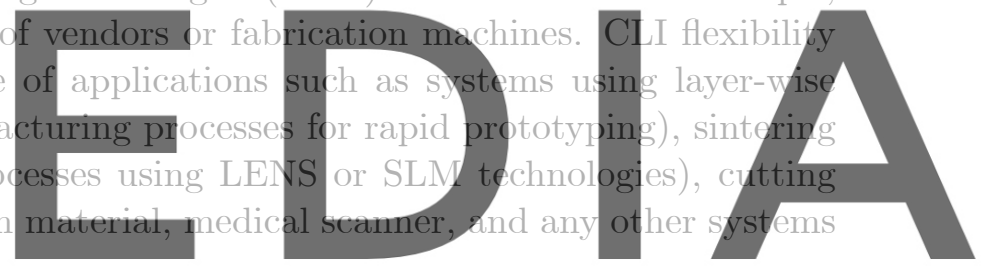

The 3D-model is sliced with parallel planes and the volume between two slices is a layer.

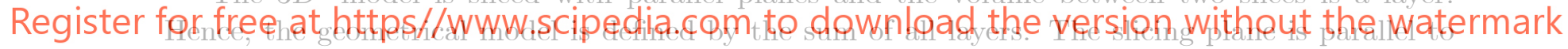

the $x y$-plane and it is assumed that the building direction is the positive $z$-axis.

Each layer is defined by its thickness and a set of contours and hatches. Contours represent the boundaries of the solid material within a layer, and are defined by polylines. Each polyline is defined by a set of point coordinates $(x, y)$, connected contiguously in the listed order by straight line segments. A hatch is a set of independent straight lines, each defined by one start and one end point. The purpose of hatches is to define filling structures to obtain a solid model.

In Table 1, an example of CLI file in ASCII-format is presented.

Both polylines and hatches are defined by coordinates of points. No reference to the time-scale is mentioned. Typically, the movement of the laser-head is defined by two parameters: the scanningspeed, $v_{M D}$, when the laser is switched-on, and the back-speed, $v_{\text {back}}$, when the laser is switched-off and it is repositioning. Hence, an automatic time-stepping $\Delta t_{o n}$ is defined by splitting each polyline (and hatch) segment according to a fix advancing step $\Delta s$ of the laser-head predefined by the enduser as a function of the dimensions of the scanning framework. When the laser is switched-off, the time-increment $\Delta t_{\text {off }}$ is computed as a function of the back-speed and the distance $\Delta x$ between the coordinates of the last scanning point (end of polyline or hatch) and the beginning of the following 


\begin{tabular}{|c|c|}
\hline (\$\$HEADERSTART & /// This is an example of CLI-format // \\
\hline$\$ \$ A S C I I$ & // File is in ASCII-format // \\
\hline$\$ \$$ UNITS $/ 1$ & $/ /$ Coordinates are given in $[\mathrm{mm}] / /$ \\
\hline$\$ \$ D A T E / 110715$ & // 11 July 2015 // \\
\hline \$LAYERS/100 & // Geometry made of 100 layers // \\
\hline \$HEADEREND & \\
\hline \$\$GEOMETRYSTART & // Start of GEOMETRY-section// \\
\hline$\$ \$ L A Y E R / 2.5$ & // Layer entry level at $z=2.5[\mathrm{~mm}] / /$ \\
\hline $\begin{array}{l}\text { \$POLYLINE/1 } 13 \\
\end{array}$ & // 3 points $(x, y)$ defining the polyline $/ /$ \\
\hline \$\$HATCHES/1 $1 \quad 7.59 .2 \quad 2.79 .2$ & // 2 points $(x, y)$ defining the hatch line // \\
\hline
\end{tabular}

Table 1: Example of CLI file in ASCII-format to define the scanning-path sequence.
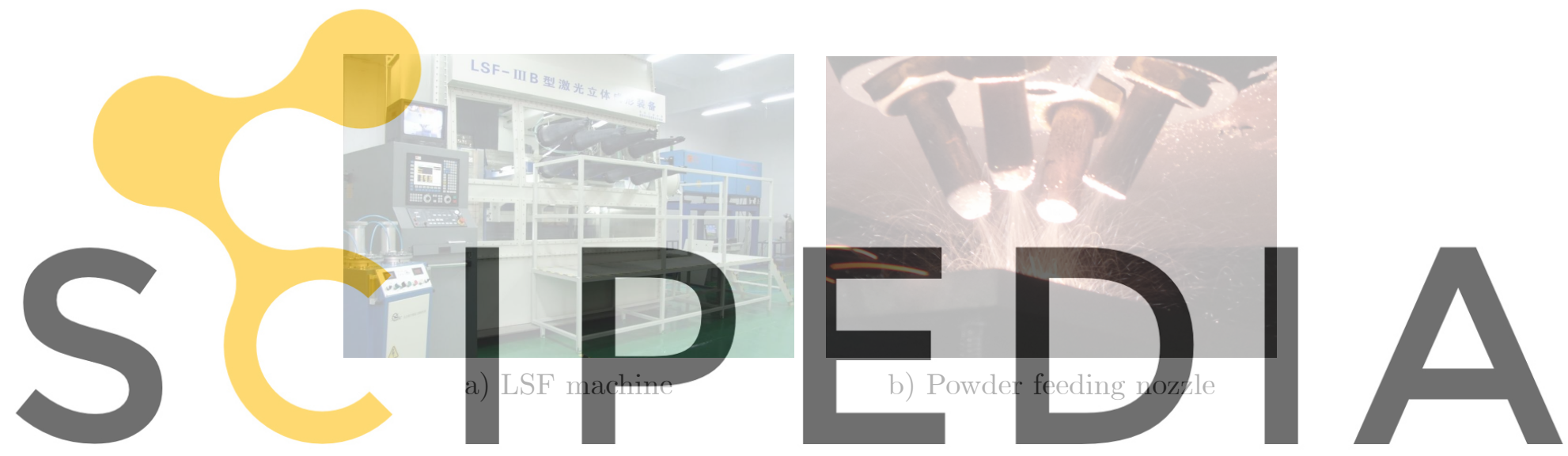

Register for free at https//www.scipedia.com to download the version without the watermark one:

$$
\begin{array}{ll}
\Delta t_{o n}=v_{M D} \Delta s & \text { During the laser scanning } \\
\Delta t_{o f f}=v_{\text {back }} \Delta x & \text { For repositioning }
\end{array}
$$

Remark 10 It is very important to include the interval $\Delta t_{\text {off }}$ as part of the thermal analysis because of the the fast cooling experienced by the material in this time-step.

\section{Experimental campaign}

The experimental campaign has been carried out at the State Key Laboratory of Solidification Processing (SKLSP) using the laser solid forming ( $L S F-I I I)$ machine shown in Figure $1 a$. The system uses a $\mathrm{CO}_{2}$-laser source with a maximum power input of $4[k W]$ in a close chamber with a protection atmosphere (argon) to prevent oxidation. Figure $1 b$ shows the coaxial nozzle used for the powder blowing. Its movement is monitored by a numerical control device.

The temperature is measured using OMEGA $G G-K-30$ thermocouples and a midi $L O G G E R$ G900-4/8 data recorder. 


\begin{tabular}{|l|r|l|}
\hline Power input & 2 & {$[\mathrm{~kW}]$} \\
\hline Laser beam size & 1.6 & {$[\mathrm{~mm}]$} \\
\hline Offset distance & 0.8 & {$[\mathrm{~mm}]$} \\
\hline Up-lift height & 0.3 & {$[\mathrm{~mm}]$} \\
\hline Scanning speed & 10 & {$[\mathrm{~mm} / \mathrm{s}]$} \\
\hline Back speed & 50 & {$[\mathrm{~mm} / \mathrm{s}]$} \\
\hline Powder feeding & 8.5 & {$[\mathrm{r} / \mathrm{min}]$} \\
\hline
\end{tabular}

Table 2: Process parameters used to inform the LSF machine.

The substrate samples consist of a Ti6Al4V Titanium alloy plates, $140[\mathrm{~mm}]$ long, $50[\mathrm{~mm}]$ wide and $6[\mathrm{~mm}]$ thick. Each plate is burnished by sand paper and cleaned using anhydrous-alcohol and acetone. Later on, 5 thermocouples are spot-welded: 3 on the lower surface and 2 more on the upper surface of the sample, as shown in Figures $2 a-b$. Finally, the substrate is clamped to the supporting system and anchored inside the chamber of the AM machine.

The Ti6Al4V powder is dried in a vacuum oven at $120\left[{ }^{\circ} \mathrm{C}\right]$ before supplying the feeding device.

Table 2 shows the parameters used to inform the control system of LSF machine.

The scanning path is shown in Figure 2c. This sequence is repeated 10 times to build 10 layers of added material. The up-lift of the nozzle is $0.3[\mathrm{~mm}]$ assuming that this is the incremental height of each layer for a fina of one of the substrat the LSF machine. Th
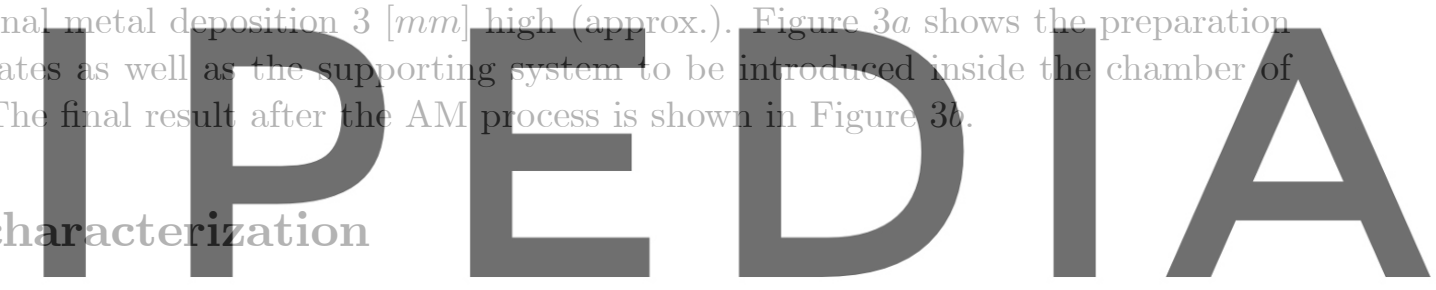

Numerical simulation of the AM process requires the characterization of the material properties to

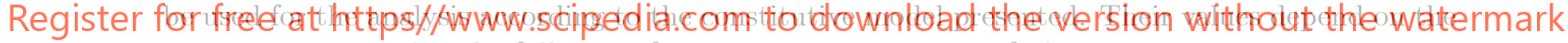

temperature, covering the full range from room temperature to fusion temperature.

In the present work, the target material is Ti6 Al4V titanium alloy for both the substrate and the powder feeding used in the AM process. Figure 4 shows the temperature-dependent properties used for this alloy within the available temperature range ([4] and [15]).

The liquidus-to-solidus phase change is defined by a latent heat of $L=290[\mathrm{~kJ} / \mathrm{kg}]$, together with the liquidus and solidus temperatures of $1703\left[{ }^{\circ} \mathrm{C}\right]$ and $1697\left[{ }^{\circ} \mathrm{C}\right]$, respectively.

The substrate is clamped to a supporting structure made of stainless steel. This structure is not analysed, even if the influence of its thermal inertia is studied to calibrate the heat loss by heat conduction through the clamping system.

\section{Numerical results}

This section describes the calibration of the in-house COupled Thermo-MEchanical FE software (COMET) customized for the numerical simulation of the AM process [8]. Computations and CPU-times refer to a labtop equipped with a $i 5$ Intel processor. 


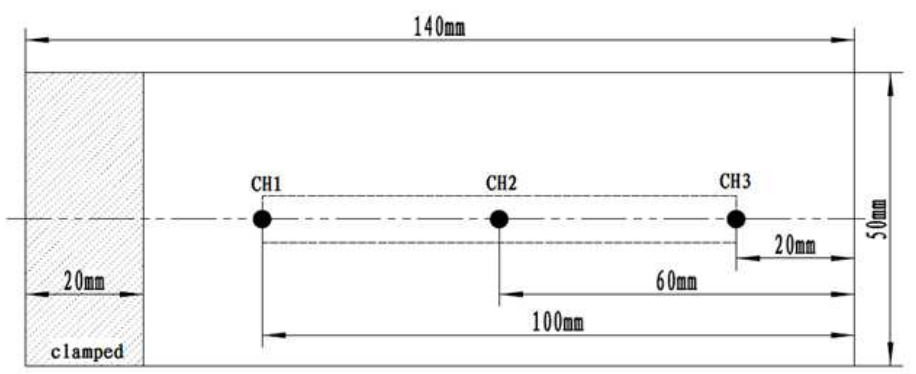

a) Lower surface

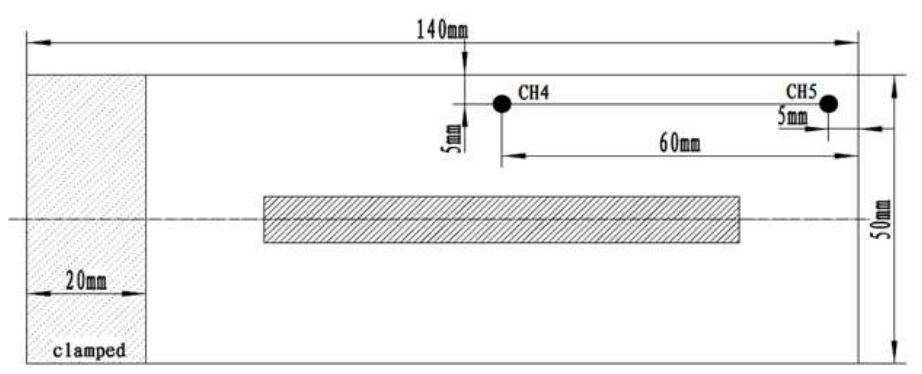

b) Upper surface

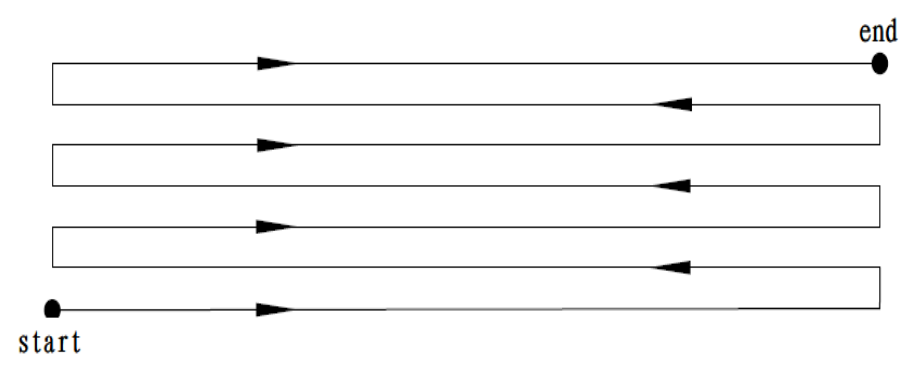

c) Scanning sequence

Figure 2: Location of thermocouples on the lower and upper surfaces of the substrate. 


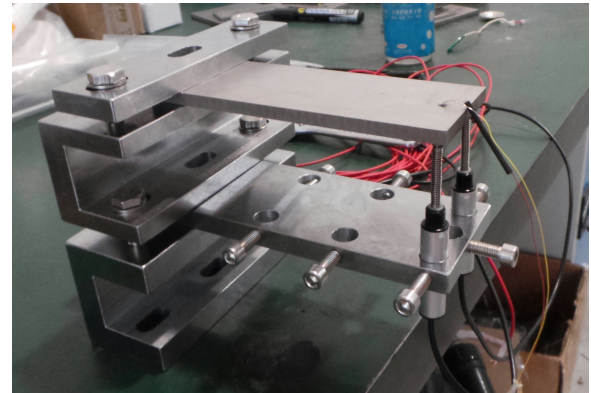

a) Substrate and supporting system

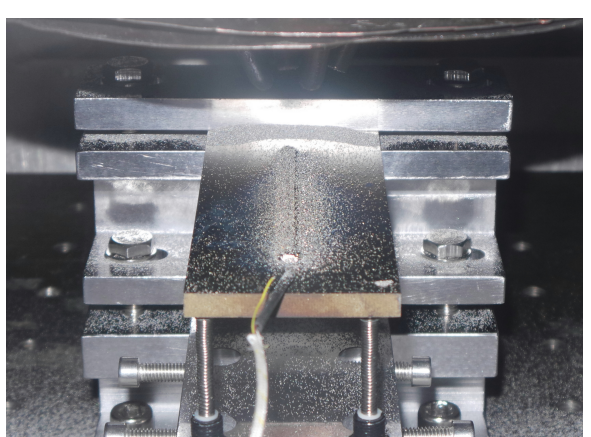

b) After the AM process

Figure 3: Substrate plate and the supporting system used for the AM process.

The results obtained through the numerical simulation are presented in terms of temperature graphs at the different thermocouple locations on the substrate, as well as temperature contour-fills, using the in-house pre-post-processor $G i D$ [17]. These results are compared with the corresponding measurements obtained at SKLSP research labs.

\subsection{Calibration and sensitivity analysis}

The calibration and sensitivity analysis have been carried out using the $F E$ discretization shown in Figure 5a. It consists of 12, 456 nodes and 10,050 hexahedral elements. The average mesh size is $2.5[\mathrm{~mm}]$, with 5 elements placed through the thickness of the plate. A finer mesh, $0.875[\mathrm{~mm}]$, is used at the process zone to be able to capture both the high temperature gradient induced by the AM process and the scanning pattern used for the metal deposition (see Figure $5 b$ ). The mesh size adopted for the metal deposition closely approximates the actual dimensions of the sinterized titanium powder. This means: $80[\mathrm{~mm}]$ long, $7[\mathrm{~mm}]$ wide $(6.4[\mathrm{~mm}]$ expected) and $2.8[\mathrm{~mm}]$ thick (3 $[\mathrm{mm}]$ expected). Hence, the average layer thickness is set to $0.28[\mathrm{~mm}]$ while the spot-size is set to $1.75[\mathrm{~mm}]$ with $50 \%$ overlapping during the scanning operation. Note that even if the expected and the actual dimensions of the metal deposition are not very different (less than 10\%), their correct values are necessary not to over-estimate the thermal inertia of the sintering material.

The colored zone in Figure $5 a$ represents the surface in contact with the clamping system used to restrain the movement of the substrate plate. Conduction heat transfer at the contact interface between the plate and the clamping system is considered. The heat transfer coefficient used for Newton's model is $50\left[\mathrm{~W} / \mathrm{m}^{2} \mathrm{~K}\right]$.

The temperature of the supporting structure is often assumed as constant during the analysis because its thermal inertia is generally much higher than the one of the sample. This was not the case in our experimental setting, so that the temperature of the clamping surface was monitored during the entire duration of the AM process and the following cooling phase. Figure $12 b$ shows the temperature evolution at the clamping system as well as the approximating curve used for the numerical simulation.

The AM process is performed in a closed chamber with a protection atmosphere to prevent 


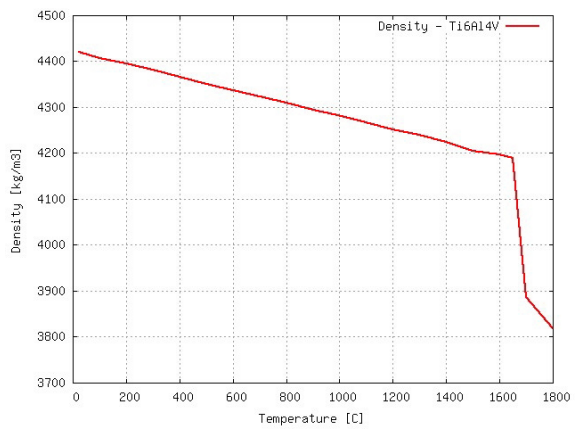

a) Density

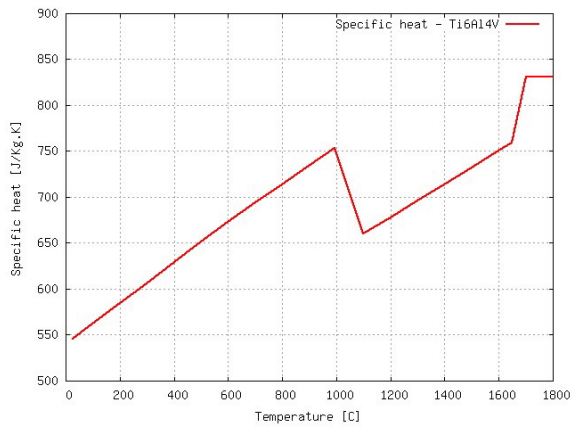

b) Specific heat

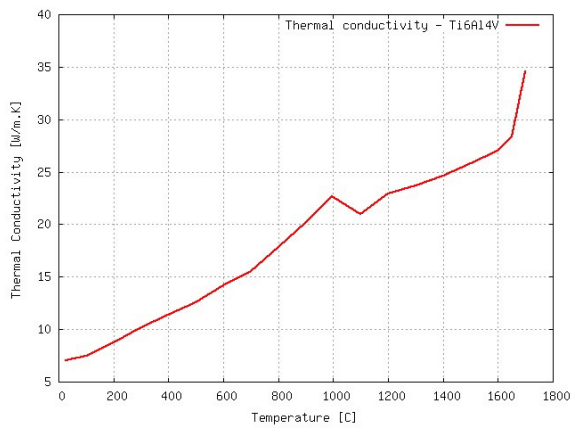

c) Thermal conductivity

Figure 4: Ti6Al4V titanium alloy: thermal material properties. 


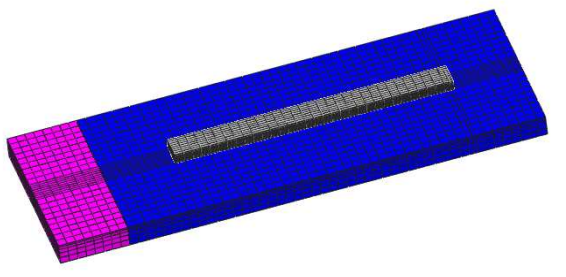

a) FE mesh

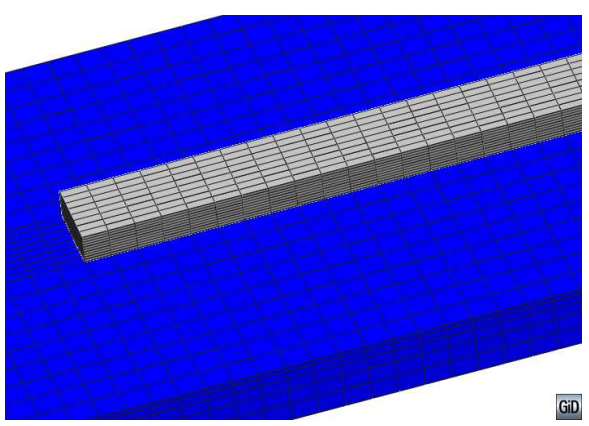

b) Detail of FE mesh at the process zone

Figure 5: FE mesh used for the numerical simulation of the AM process.

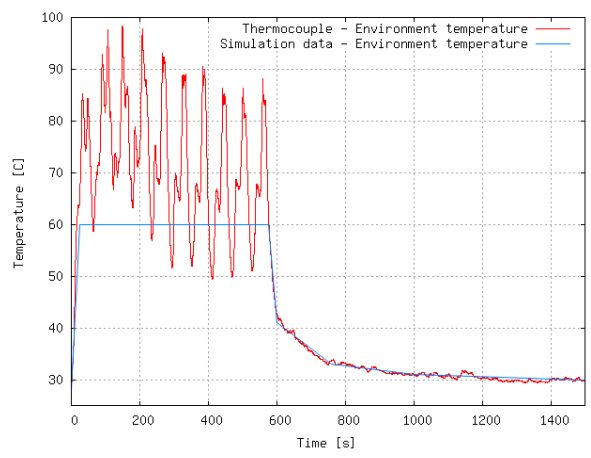

a) Environment temperature

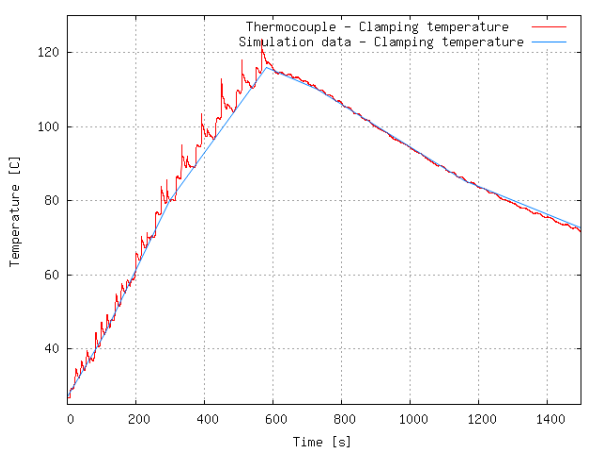

b) Temperature at the clamping system

Figure 6: Actual temperature of the environment (inside the AM chamber) and at the clamping system. Measured values and simplified evolution for the numerical model. 


\begin{tabular}{|l|r|l|}
\hline \hline Power & 2 & {$[\mathrm{~kW}]$} \\
\hline Power absorption & 16.5 & {$[\%]$} \\
\hline Scanning speed & 10 & {$[\mathrm{~mm} / \mathrm{s}]$} \\
\hline Back speed & 50 & {$[\mathrm{~mm} / \mathrm{s}]$} \\
\hline Penetration & 0.28 & {$[\mathrm{~mm}]$} \\
\hline Layer thickness & 0.28 & {$[\mathrm{~mm}]$} \\
\hline Layer width & 1.75 & {$[\mathrm{~mm}]$} \\
\hline Overlapping & 50 & {$[\%]$} \\
\hline
\end{tabular}

Table 3: Process parameters used for the numerical simulation.

oxidation. Also in this case, the environment temperature was not constant. Figure $12 a$ shows the actual temperature evolution inside the chamber. Unfortunately, the thermocouple was placed to close to the process area, being affected by the heat radiation during AM process. The (conservative) environment temperature value used for both radiation and convection models is shown in Figure $12 a$ (blue line). The HTC coefficient used for the heat convection model is $10\left[\mathrm{~W} / \mathrm{m}^{2} \mathrm{~K}\right]$ while emissivity coefficient used for the heat radiation model is 0.7 .

Table 3 shows the process parameters used for the numerical simulation. These parameters are slightly different of the experimental ones according to the actual size of the metal deposition.

Figures $7 a-b$ show both measured and calculated temperatures at the lower and upper surfaces, respectively. The agreement is remarkable. A closer view is presented in Figures $7 c-d$ where it is possible to appreciate the accuracy of the numerical results.

Remark 11 Thermocouples 4 and 5, placed on the upper surface of the substrate, were shielded to protect them against radiation. Nevertheless, the recorded temperatures still suffer from the heat radiation effects.

The simulation strategy consists of 3 phases: the pre-heating phase, the AM phase and, finally, the cooling phase. During the simulation of the manufacturing phase, the power absorption is set to $16.5 \%$, meaning that most of the energy input is scattered in the surrounding environment, in agreement with the experimental experience at $S K L S P$. Figures $8 a-b$ show the sensitivity to the power absorption parameter. This is the most important parameter of the balance of energy equation in (4) defining the energy input into the system. During the pre-heating phase, the absorption parameter is reduced to $10 \%$. This phase is not contemplated in the experimental setting, but it can be clearly observed that the powder sintering is much less during the scanning sequence defined for the first layer. This is due to the low temperature of the substrate in this first phase and it can also explain the mismatch between the estimated and the actual height of the metal deposition (0.2 [ $\mathrm{mm}]$ approx.).

Figure $9 a$ and the corresponding detailed view in Figure $9 b$ show the temperature evolution at thermocouple 2 and its sensitivity to the pre-heating phase.

Remark 12 The benchmark proposed for the calibration of the numerical model only considers 10 layers for metal deposition, highlighting the difference between the pre-heating and the manufacturing phases. In the case of much higher depositions, the pre-heating phase can be neglected. 


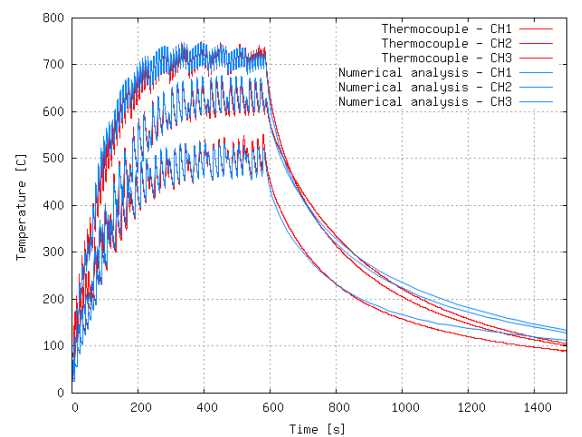

a) Thermocouples placed on the lower surface of the substrate

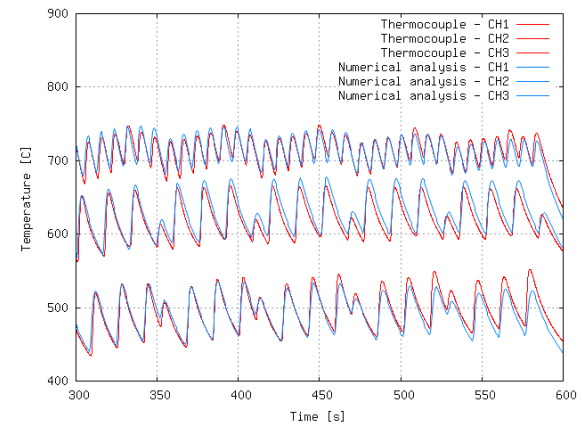

c) Detail of thermocouples $\mathrm{CH} 1$, $\mathrm{CH} 2$ and $\mathrm{CH} 3$

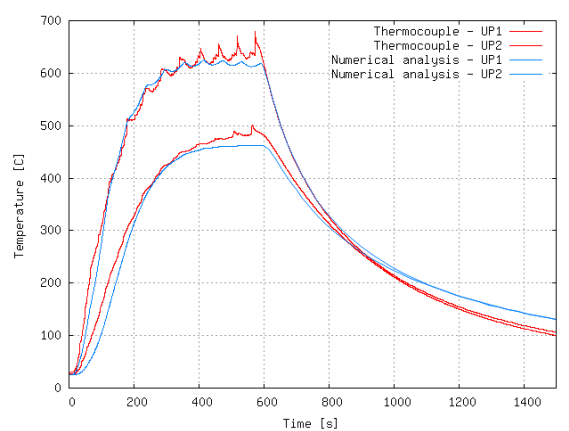

b) Thermocouples placed on the upper surface of the substrate

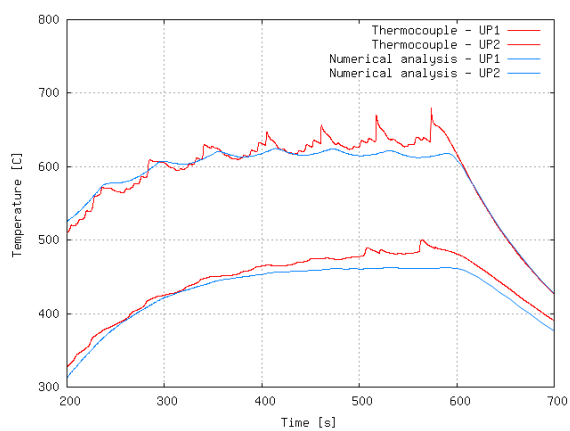

c) Detail of thermocouples $\mathrm{CH} 4$ and $\mathrm{CH} 5$

Figure 7: Temperature evolution at 5 thermocouple locations: 3 placed on the lower surface of the substrate (CH1, CH2 and $\mathrm{CH} 3)$ and 2 on the upper surface (CH4 and $\mathrm{CH} 5)$. Comparison between the experimental evidence and the numerical model propoed. 


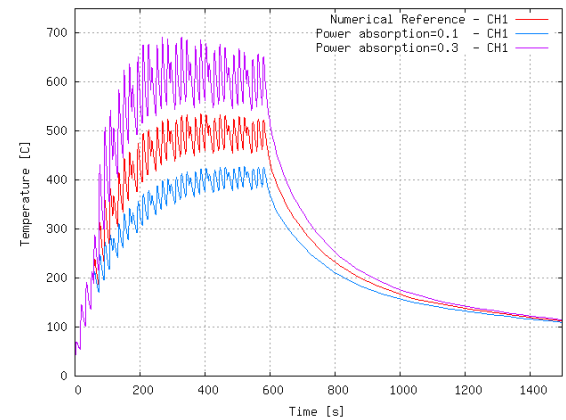

a) Temperature evolution at $\mathrm{CH} 1$

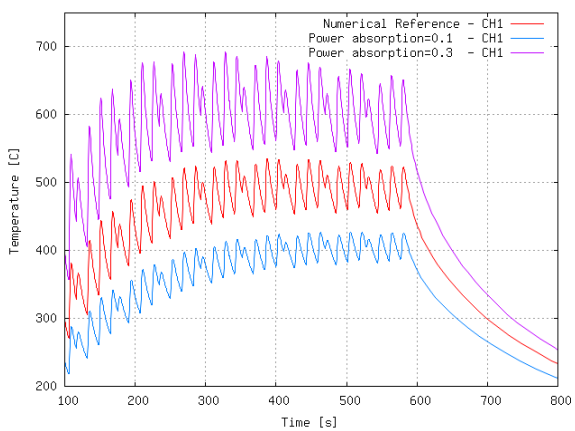

b) Detail

Figure 8: Sensitivity to the power absorption parameter.

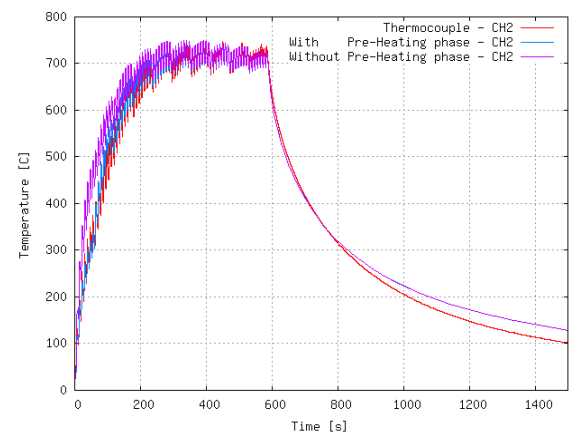

a) Temperature evolution at $\mathrm{CH} 2$

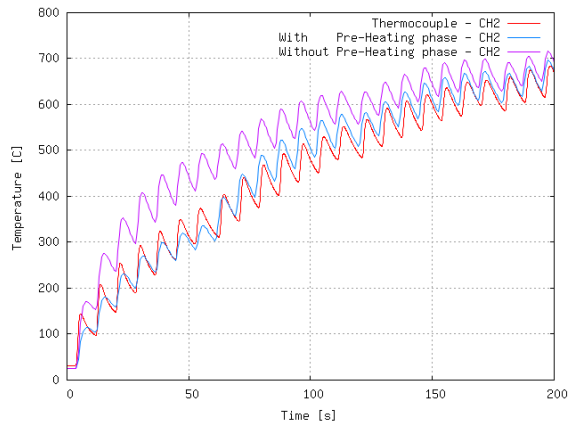

b) Detail

Figure 9: Effect of the pre-heating phase. Comparison between the measured temperature evolution at thermocouple $\mathrm{CH} 2$ and the numerical simulation.

Figures $10 a-b$ present the thermal response at thermocouples 1 and 2, respectively, comparing LENS vs. SLS technologies. Even if the two manufacturing processes (and corresponding machines) are quite different, from the simulation point of view, the results are very similar. In the case of the LENS technology, the power beam is focussed on the substrate surface where the molten-pool is generated. The focus is moved upward to the powder bed surface for the SLS technology.

During the AM process, both the substrate and the powder material undergo the phase change: on the one hand, the enegy input generates the melting pool and melts the blown powder; on the other hand, the material is sinterized, forming a new layer by the coalescence process. Nevertheless, both the heating and the cooling phases are very fast and, the latent heat is firstly absorbed (melting phase) and later, is released (sintering phase). Hence, the effect of the latent heat contribution at global level is negligible as shown in Figures 11a-b. All the corresponding curves are perfectly overlapping. This result is very interesting because, without considering the phase-change, the 


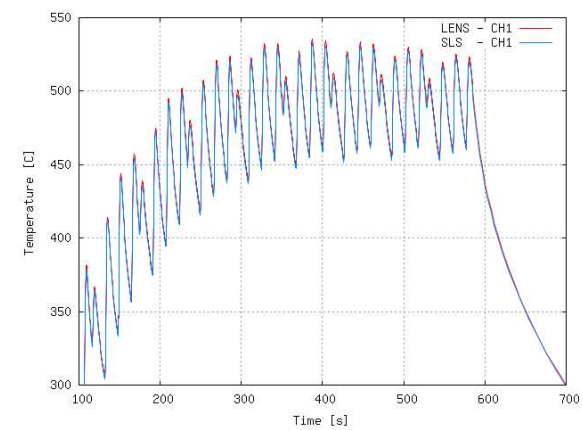

a) $\mathrm{CH} 1$

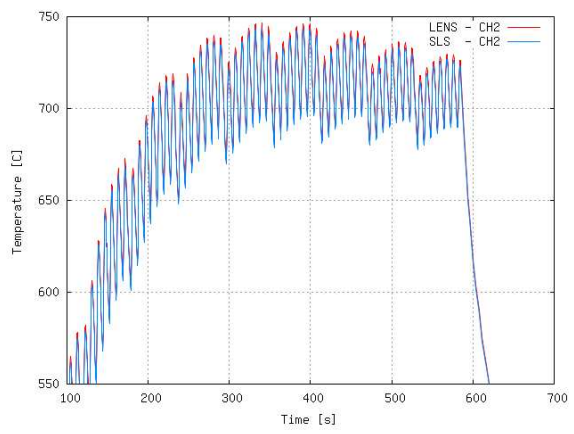

b) $\mathrm{CH} 2$

Figure 10: LENS vs. SLS technology: numerical results at CH1 and CH2.

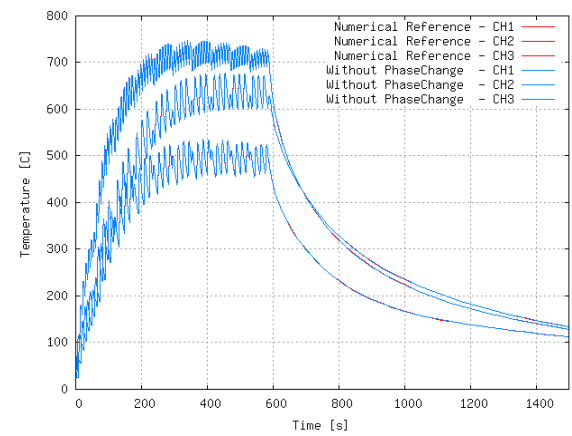

a) Temperature evolution: CH1-CH3

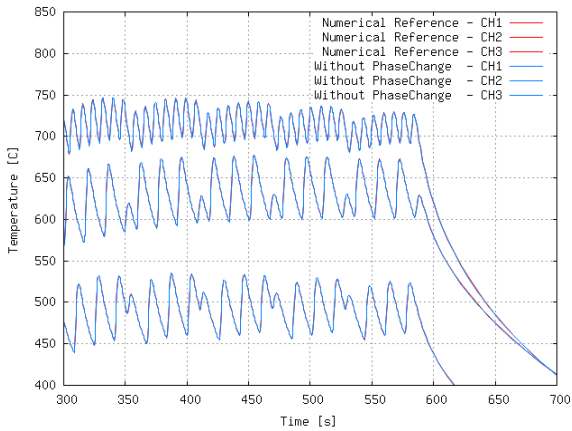

b) Detail

Figure 11: Effect of fusion phase-change contribution.

CPU-time is reduced by 3 times ( 1 iteration instead of 3-4 during the phase-change) making the numerical simulation of the AM process much more affordable. Table 5 shows the CPU-time of the first simulation proposed (used as reference) and the case where the phase-change is neglected.

In the following, the sensitivity to the heat loss models is presented. This is the key point when studying the AM simulation process. The equilibrium between the energy absorption and the heat dissipated through the surrounding environment defines the temperature evolution in the structure. To uncouple the two mechanisms, the heat loss has been calibrated analysing the cooling phase, when the energy input is switched-off. From the physical point of view, both heat convection and heat radiation coexist but it is difficult to split their effects. In this work, the reference solution is obtained calibrating the heat radiation model, while keeping the HTC of the convection model $\left(h_{\text {cond }}\right.$ ) to a constant value of $10\left[\mathrm{~W} / \mathrm{m}^{2} \mathrm{~K}\right]$. The resulting emissivity coefficient used for the heat radiation model was: $\varepsilon_{r a d}=0.7$. Figures $12 a$ shows the response of the numerical model when using the heat convection model, only. The result is wrong from both the quantitative and qualitative 


\begin{tabular}{|c|c|}
\hline \hline Temperature $\left[{ }^{\circ} \mathrm{C}\right]$ & HTC $\left[\mathrm{W} / \mathrm{m}^{2} \mathrm{~K}\right]$ \\
\hline \hline 20 & 6 \\
\hline 200 & 10 \\
\hline 400 & 20 \\
\hline 600 & 40 \\
\hline 800 & 70 \\
\hline 1000 & 110 \\
\hline 1200 & 160 \\
\hline
\end{tabular}

Table 4: Best suited HTC for the convection model.

points of view, either in the cooling or heating phases. In fact, even if the HTC is increased (or the power absorption is reduced) to capture the maximum temperature level, the temperature evolution is still far from the experimental evidence. More specifically, the cooling phase is always too slow, while the heating phase never shows the temperature saturation experimented by the material, as shown in Figure 12b. Figure 12c presents the temperature evolution at the thermocouple 2, when the heat radiation model is used for the metal deposition layers, only. Once again, it is demonstrated that the main mechanism to dissipate the heat from the substrate is by radiation ([19]). Table 4 shows the values of $h_{\text {loss }}(T)$ when using a temperature-dependent HTC for the total heat loss as presented in Eq. (12). This model (not shown) gives exactly the same solution as the numerical reference. Finally, the response of the numerical model, when a linear interpolation of the HTC vs. temperature is assumed, is presented in Figure 12d. If a maximum value of 160 $\left[\mathrm{W} / \mathrm{m}^{2} \mathrm{~K}\right]$ is adopted, the temperature curves saturate to very low values. Instead, when using 90 $\left[W / m^{2} K\right]$ as top value, the response compares very well to the reference one.

Figure $13 a$ shows the sensitivity of the model to the temperature of the surrounding environment. The difference is negligible when the radiation model is used, while a small mismatch can be observed when only the convection model is adopted, as shown in Figure $13 b$.

The most suitable heat loss mechanism at the contact interface between the substrate and the clamping system is by heat conduction, using the Newton's law in Eq. (7). Also in this case, the HTC as well as the temperature of the clamping system must be defined. In Figure $14 a$ it is demonstrated that the differences due to the temperature defined at the clamping interface are almost negligible during the heating phase. An appreciable discrepancy is shown during the cooling phase. Using a constant value of $25^{\circ} \mathrm{C}$, the final equilibrium temperature is lower than expected because of the heating of the supporting structure. More important is the definition of the HTC by conduction: this is the inverse of the thermal resistivity at the contact interface. Its value depends on the roughness of the surfaces in contact as well as on the contact pressure established. In our case, the most suitable value was $50\left[\mathrm{~W} / \mathrm{m}^{2} \mathrm{~K}\right]$, being far from the typical value of $1000\left[\mathrm{~W} / \mathrm{m}^{2} \mathrm{~K}\right]$ when the contact is almost perfect. This can be explained by the fact that the bolts torque was very low because applied just manually. Figure $14 b$ shows the model sensitivity to the HTC value, compared to the calibrated numerical reference.

Some considerations about the time-stepping used are necessary. The calibrated solution makes use of an automatic time-step which moves the heat source element-by-element (Courant's number: $C u=1)$. This allows for the most accurate and more CPU-time consuming result. The time-step 


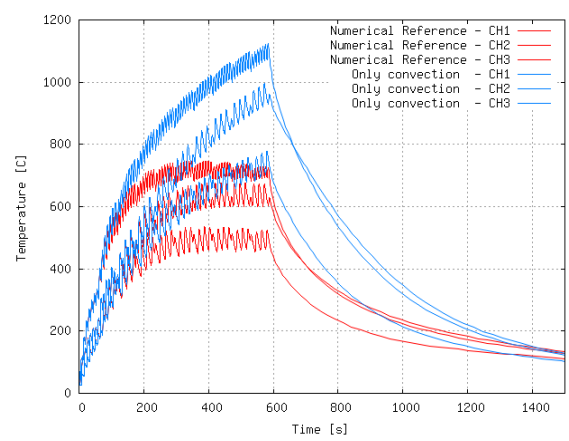

a) Radiation vs. convection models

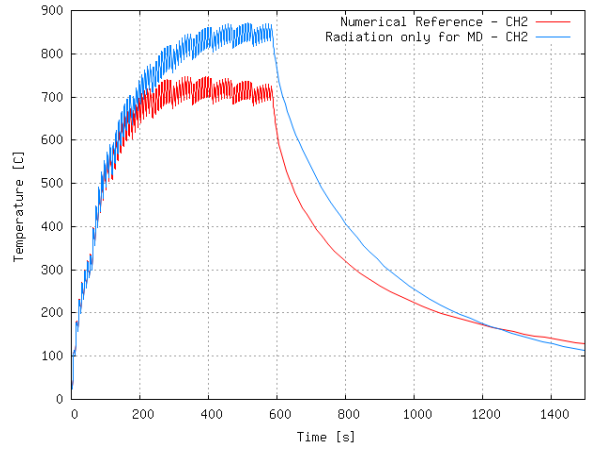

c) Heat radiation only for the deposited layers.

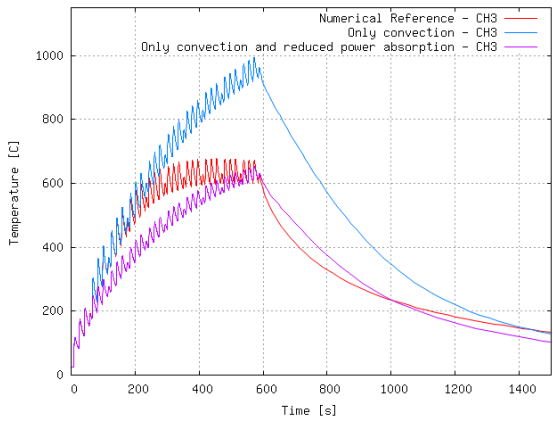

b) Convection model with reduced power input

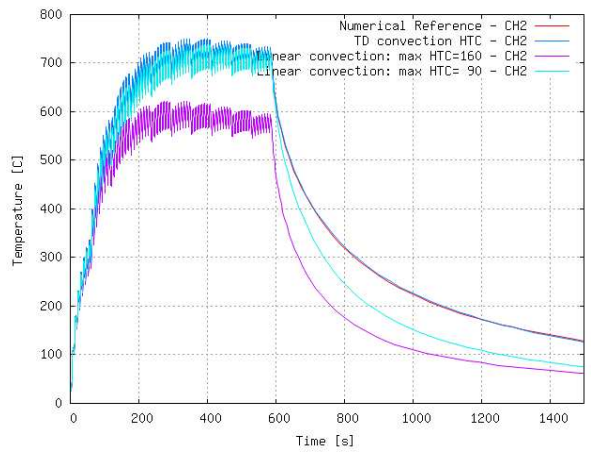

d) Linear convection HTC

Figure 12: Effects of the heat loss by radiation. Comparison between radiation and convection models. 


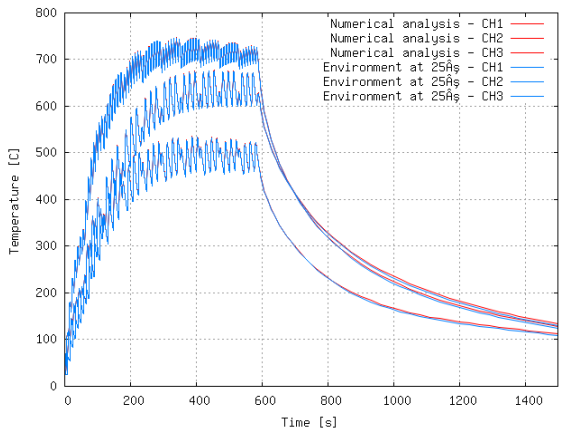

a) Effect of the environment temperature. Measured vs. constant value of $25^{\circ} \mathrm{C}$

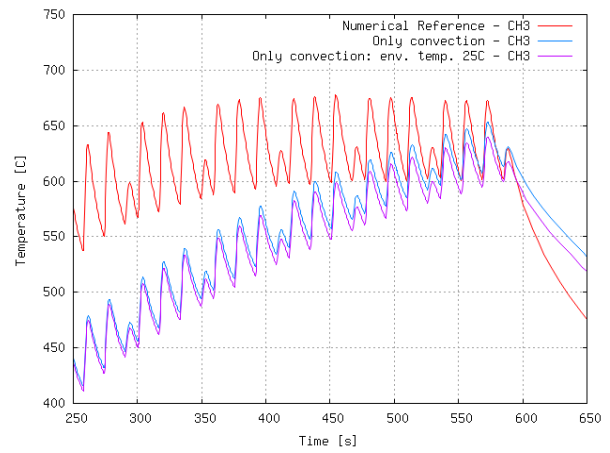

b) Effect of the environment temperature when using only convection model

Figure 13: Effect of the actual temperature of the environment (measured inside the AM chamber) on the response of both radiation and convection models.

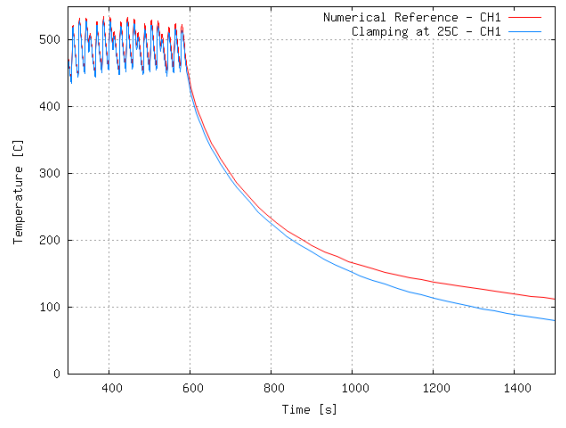

b) Effect of the temperature at the clamping system. Measured vs. constant value of $25^{\circ} \mathrm{C}$

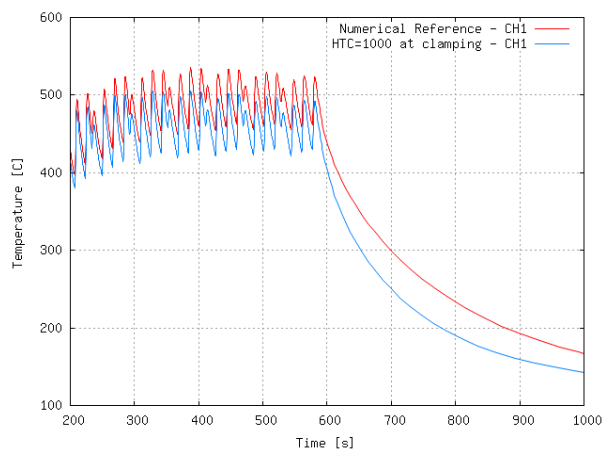

b) Comparison between the best suited HTC for the heat conduction model and typical value used for perfect heat transmission

Figure 14: Effect of the HTC and the actual temperature measured at the clamping system used for the heat conduction model at the contact interface between the substrate and the supporting structure. 


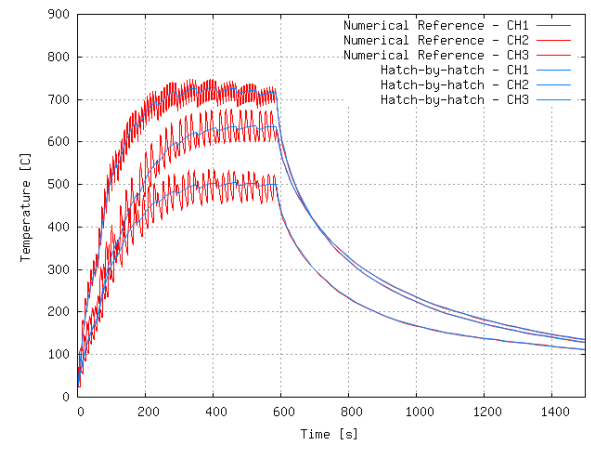

a) Temperature evolution at lower thermocouple locations

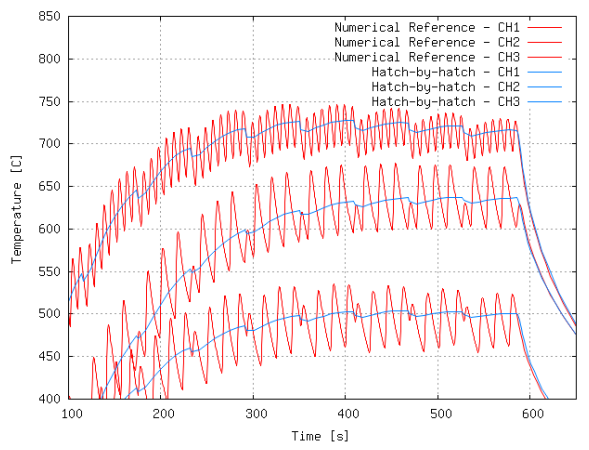

b) Detail

Figure 15: Metal deposition built-up hatch-by-hatch. 7 hatches for each layer.

\begin{tabular}{|l|c|c|}
\hline \hline Strategy & CPU-time $[s]$ & {$[\%]$} \\
\hline \hline Reference & 1356 & 100 \\
\hline No phase-change & 473 & 35 \\
\hline Hatch-by-hatch & 61 & 4 \\
\hline Layer-by-layer & 29 & 2 \\
\hline
\end{tabular}

Table 5: Simulation CPU-time using different strategies.

can be increased at the cost of reducing the simulation accuracy. One interesting option was to study the response of the numerical model when all the elements belonging to one scanning hatch are activated simultaneously. This means 7 time-steps, and the corresponding cooling intervals after the activation of each hatch, for each deposited layer. The result is presented in Figures 15a-b. It is interesting to observe that, even if the hatch-by-hatch activation loses the oscillatory response of the temperature, it conserves its average value. Clearly, the CPU-time is notably reduced, as show in Table 5.

Finally, the activation sequence could be performed in a layer-by-layer manner. In each timestep, all the elements belonging to one layer are activated adopting an equivalent scanning speed of $1.4[\mathrm{~mm} / \mathrm{s}]$. The result is presented in Figures $16 a-b$. Once again, the balance of energy allows for an average estimation of the temperature profiles while losing all the details concerning the scanning sequence.

Remark 13 Even if both hatch-by-hatch and layer-by-layer activation sequences can appear promising, because of the CPU-time reduction, the mechanical response can be compromised when performing the stress analysis. In fact, the final microstructure, the mechanical properties, the plastic deformations and the residual stresses are influenced by the complex thermal history (local heating and cooling) induced by the scanning pattern.

Figure $17 a$ shows the sample after cooling at room temperature with the mark induced by the 


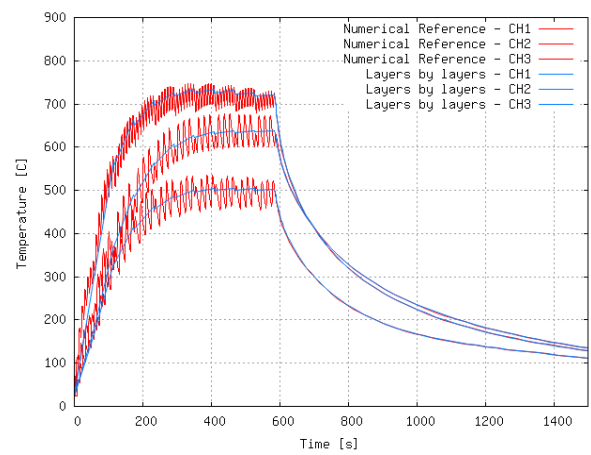

a) Temperature evolution at lower thermocouple locations

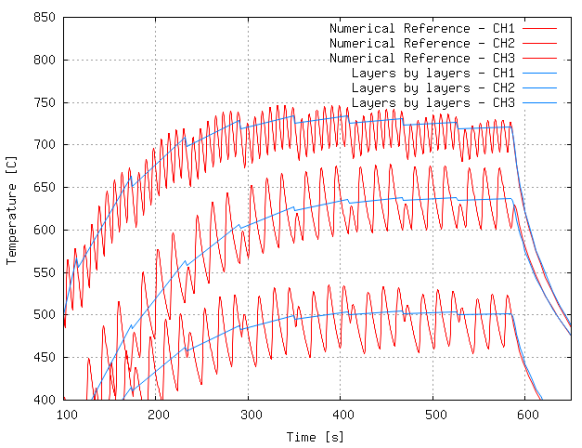

b) Detail

Figure 16: Metal deposition built-up layer-by-layer. One time-step for each layer.

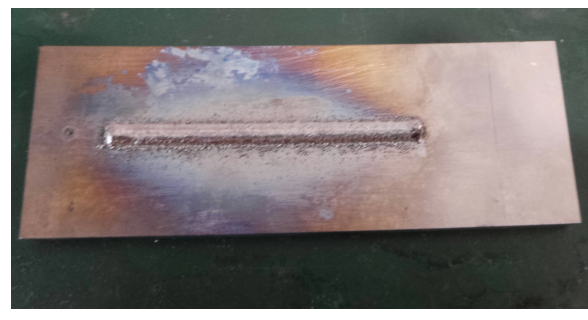

a) Oxidation after AM process

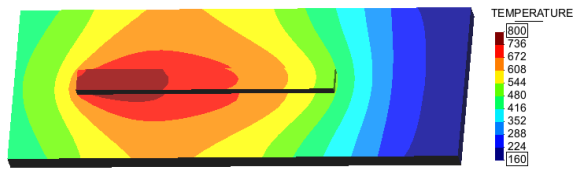

b) Temperature contour-fill at the end of the AM process

Figure 17: Temperature contour-fill at the end of the manufacturing process. Comparison with the residual sample mark.

red-hot temperature field. Figure $17 b$ shows the temperature contour-fill at the end of the AM process just before cooling. Once again the agreement between them is remarkable.

\section{Conclusion}

In this work, a FE framework for the numerical simulation of the AM process is presented, detailing the mechanisms of energy absorption and heat dissipation through the surrounding environment. The suitable FE activation technique is also discussed, focussing on the numerical simulation of fabrication processes by blown powder technology.

The experimental work has been carried out at SKLSP laboratories using a blown powder (LSF-III) machine.

The calibration of the numerical model has demonstrated a remarkable agreement with the experimental evidence either during the deposition process or the following cooling phase to the 
room temperature.

This work has also studied the sensitivity to different process parameters and modeling data. The outcome is the following:

- The model is very sensitive to the energy absorption parameter. In the case of blown powder technology, this parameter can be adjusted around 15\%. A slightly lower value (11\%) can be considered during the scanning of the first layer (pre-heating phase).

- Heat radiation is the main mechanism to dissipate heat to the surrounding environment. It cannot be neglected, but it can be replaced by a temperature-dependent HTC. The temperature variation inside the process chamber or at the clamping system can be neglected. Nevertheless, better accuracy can be achieved in the cooling phase if those data are available.

- The latent heat contribution of the liquidus-to-solidus phase-change can be neglected, saving $60 \%$ of the total CPU-time. Its effect at global level is negligible.

- The simulation time can be speeded-up by considering a hatch-by-hatch or even a layer-bylayer deposition sequence. Average temperature fields can be recovered but the accuracy on the local thermal history is lost.

\section{Acknowledgments}

Financial support from the EC - Factories of the Future (FoF) Program under the CA×Man Project - Computer Aided Technologies for Additive Manufacturing - within Horizon 2020 Framework Programme and funding from the Spanish Ministry of Economy and Competitivity under the EACY project (Ref. MAT2013-48624-C2-1-P) - Enhanced accuracy computational and experimental framework for strain localization and failure mechanisms - within the Excellency Programme for Knowledge Generation are gratefully acknowledged.

This research work is also supported by the National Natural Science Foundation of China (Grants 51323008 and 51271213[]), the National Basic Research Program of China (Grants 2011CB610402), the National High Technology Research and Development Program of China (Grant 2013AA031103), the Specialized Research Fund for the Doctoral Program of Higher Education of China (Grant No. 20116102110016) and the State Administration of Foreign Experts Affairs of China through the High-end Experts Recruitment Program.

\section{References}

[1] Agelet de Saracibar C., Cervera M. and Chiumenti M., On the formulation of coupled thermoplastic problems with phase-change, Int. Journal of Plasticity, 15 (1999) 1-34.

[2] Aggarangsi P, Beuth J, Griffith M. Melt pool size and stress control for laser-based deposition near a free edge. Proc. solid freeform fab symp. The University of Texas at Austin, (2003) 196-207.

[3] Anca A, Fachinotti V, Escobar-Palafox G, Cardona A. , Computational modelling of shaped metal deposition. Int J Numer Methods Eng 85(1) (2011) 84-106. 
[4] Bermingham M.J. , McDonald S.D. , Dargusch M.S. , StJohn D.H. , Microstucture of Cast Titanium Alloys, Materials Forum, 31 (2007).

[5] Cervera M., Agelet de Saracibar C. and Chiumenti M., Thermo-mechanical analysis of industrial solidification processes, Int. Journal for Num. Methods in Engineering, 46 (1999) 1575-1591.

[6] Bugeda G., Cervera M., Lombera G., Oñate E., Numerical analysis of stereolithography processes using the finite element method, Rapid Prototyping Journal, 1 (2) (1995) 13-23.

[7] Bugeda G., Cervera M., Lombera G. Numerical prediction of temperature and density distributions in selective laser sintering processes, Rapid Prototyping Journal, 5(1) (1999) 21-26.

[8] Cervera M., Agelet de Saracibar C. and Chiumenti M., COMET: COupled MEchanical and Thermal analysis. Data Input Manual, Version 5.0, Technical report IT-308, htpp://www.cimne.upc.es, 2002.

[9] Chiumenti M., Agelet de Saracibar C. and Cervera M., On the Numerical Modelling of the Thermo-Mechanical Contact for Metal Casting Analysis, Journal of Heat Transfer, 130 (2008) $1-10$.

[10] Chiumenti M., Cervera M., Salmi A., Agelet de Saracibar C., Dialami N., Matsui K., Finite element modeling of multi-pass welding and shaped metal deposition processes. Computer Method Appl Mech Eng 199 (2010), 2343-2359; DOI: 10.1016/j.cma.2010.02.018.

[11] Darmadi D. B., Validating the Accuracy of Heat Source Model via Temperature Histories and Temperature Field in Bead-on-plate Welding, Int. J. of Eng. \& Technology, 11(5) (2011) 505-686.

[12] Deng D., Murakawa H., Numerical simulation of temperature field and residual stress in multipass welds in stainless steel pipe and comparison with experimental measurements, Computational Materials Science 37 (2006) 269-277.

[13] Deng D., Murakawa H., Liang W., Numerical simulation of welding distortion in large structures, Comput. Methods Appl. Mech. Engrg. 196 (2007) 4613-4627.

[14] Denlinger E, Heigel J, Michaleris P. Residual stress and distortion modelling of electron beam direct manufacturing Ti-6Al-4V. Proc Inst Mech Eng B:J Eng Manuf. 11 (2014)

[15] Elmer J. W., Palmer T. A., Babu S.S., Zhang W., and DebRoy T., Phase transformation dynamics during welding of Ti-6Al-4V, Journal of Applied Physics, n. 12, 95 (2004), DOI: 10.1063/1.1737476.

[16] Gajapathi S.S., Mitra S. K. and Mendez P. F., Controlling heat transfer in micro electron beam welding using volumetric heating, International Journal of Heat and Mass Transfer, 54 (2011) 5545-5553.

[17] GiD: The personal pre and post preprocessor. htpp://www.gid.cimne.upc.es, 2002. 
[18] Goldak J., Chakravarti A., Bibby M., A new finite element model for welding heat sources, Metall. Trans. 15B (1984) 299-305.

[19] Heigel J.C., Michaleris P., Reutzel E.W., Thermo-mechanical model development and validation of directed energy deposition additive manufacturing of $\mathrm{Ti}-6 \mathrm{Al}-4 \mathrm{~V}$, Additive Manufacturing 5 (2015) 9-19.

[20] Huang W.D. and Lin X. Research progress in laser solid forming of high - performance metallic components at the state key laboratory of solidification processing of China, 3D Printing and Additive Manufacturing, 1(3) (2014) 156-165

[21] Jendrzejewski R, Sliwinski G, Krawczuk M, Ostachowicz W., Temperature and stress fields induced during laser cladding, Computer and Structures, 82(7) (2004) 653-8.

[22] Jendrzejewski R, Sliwinski G. Investigation of temperature and stress fields in laser cladded coatings. Appl Surf Sci, 254(4) (2007) 921-5.

[23] Kelly S, Kampe S. Microstructural evolution in laser-deposited multi-layer Ti-6Al-4V builds: Part II. Thermal modeling, Metall. Mater Trans A 35(6) (2004) 1869-79.

[24] Labudovic M, Hu D, Kovacevic R. A three dimensional model for direct laser metal powder deposition and rapid prototyping. J Mater Sci, 38(1) (2003) 35-49.

[25] Lacki P. and Adamus K., Numerical simulation of the electron beam welding process, Computers and Structures, 89 (2011), 977-985.

[26] Marimuthu S, Clark D, Allen J, Kamara A, Mativenga P, Li L, et al. Finite element modelling of substrate thermal distortion in direct laser additive manufacture of an aero-engine component. Proc Inst Mech Eng C: J MechEng Sci 227(9) (2013) 1987-99.

[27] Rahman M., Maurer W., Ernst W., Rauch R. and Enzinger N., Calculation of hardness distribution in the HAZ of micro-alloyed steel, Weld World, 58 (2014), 763-770, DOI: 10.1007/s40194014-0156-5.

[28] Rosenthal D., Mathematical theory of heat distribution during welding and cutting, Welding J. 20 (5) (1941) 220-234.

[29] Tan H., Chen J., Zhang S.Y., Lin X. and Huang W. D., Process analysis for laser solid forming of thin-wall structure. International Journal of Machine Tools \& Manufacture, 50(1) (2010) $1-8$.

[30] Tan H., Chen J., Zhang S.Y., Lin X. and Huang W. D., Estimation of laser solid forming process based on temperature measurement. Optics and Laser Technology, 42(1) (2010) 4754 .

[31] Tian Y., Wang C., Zhu D. and Zhou Y., Finite element modeling of electron beam welding of a large complex $\mathrm{Al}$ alloy structure by parallel computations, Journal of materials processing technology, 199 (2008) 41-48. 
[32] Wang L, Felicelli S, Pratt P. Residual stresses in LENS-deposited AISI 410 stainless steel plates. Mater Sci Eng A: Struct 496(1) (2008) 234-41.

[33] Wei L., Lin X., Wang M. and Huang W.D. Orientation selection of equiaxed dendritic growth by three-dimensional cellular automaton model. Physica B: Condensed Matter, 407(13) (2012) $2471-2475$

[34] Wei L., Lin X., Wang M. and Huang W.D. Cellular automaton simulation of the molten pool of laser solid forming process. Acta Physica Sinica, 64(1) (2015) 018103

[35] Yang Y-P, Babu S., An integrated model to simulate laser cladding manufacturing process for engine repair applications. Weld World, 54(9-10) (2010) R298-307.

[36] Zhang S.Y., Lin X., Chen J. and Huang W. D. Heat-treated microstructure and mechanical properties of laser solid forming Ti-6Al-4V alloy. Rare Metals, 28(6) (2009) 537-544

[37] Zekovic S, Dwivedi R, Kovacevic R., Thermo-structural finite element analysis of direct laser metal deposited thin-walled structures. Proc solidfreeform fab symp. The University of Texas at Austin, (2005) 338-55. 